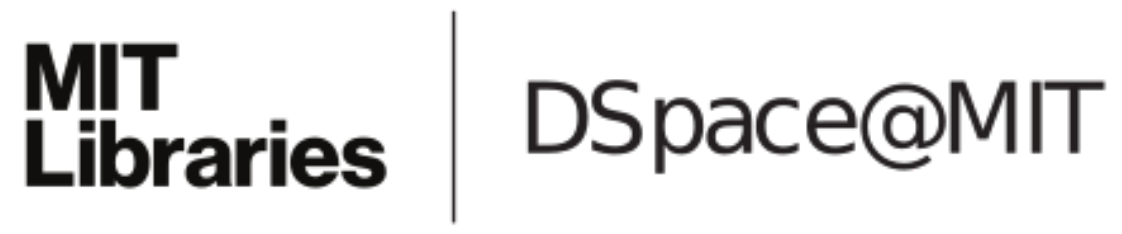

\author{
MIT Open Access Articles
}

Physical Basis of Metal-Binding Specificity in Escherichia coli NikR

The MIT Faculty has made this article openly available. Please share how this access benefits you. Your story matters.

Citation: Phillips, Christine M., Paul S. Nerenberg, Catherine L. Drennan, and Collin M. Stultz. "Physical Basis of Metal-Binding Specificity in Escherichia coli NikR." Journal of the American Chemical Society 131, no. 29 (July 29, 2009): 10220-10228.

As Published: http://dx.doi.org/10.1021/ja9026314

Publisher: American Chemical Society (ACS)

Persistent URL: http://hdl.handle.net/1721.1/82042

Version: Author's final manuscript: final author's manuscript post peer review, without publisher's formatting or copy editing

Terms of Use: Article is made available in accordance with the publisher's policy and may be subject to US copyright law. Please refer to the publisher's site for terms of use. 


\title{
Physical Basis of Metal-Binding Specificity in Escherichia coli NikR
}

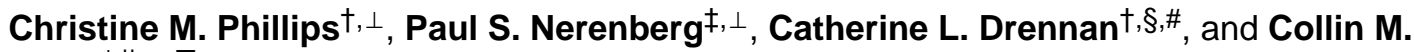 \\ Stultz ${ }^{*} \|, \perp, \nabla$ \\ Departments of Chemistry, Physics, Biology, and Electrical Engineering and Computer Science, \\ Research Laboratory of Electronics, Howard Hughes Medical Institute, and Harvard-MIT Division \\ of Health Sciences and Technology, Massachusetts Institute of Technology, Cambridge, \\ Massachusetts 02139
}

\section{Abstract}

In Escherichia coli and other bacteria, nickel uptake is regulated by the transcription factor NikR. Nickel binding at high-affinity sites in $E$. coli NikR ( $E c N i k R)$ facilitates $E c N i k R$ binding to the nik operon, where it then suppresses transcription of genes encoding the nickel uptake transporter, NikABCDE. A structure of the EcNikR-DNA complex suggests that a second metal-binding site is also present when NikR binds to the nik operon. Moreover, this co-crystal structure raises the question of what metal occupies the second site under physiological conditions: $\mathrm{K}^{+}$, which is present in the crystal structure, or $\mathrm{Ni}^{2+}$, which has been proposed to bind to low- as well as highaffinity sites on $E c N i k R$. To determine which ion is preferred at the second metal-binding site and the physical basis for any preference of one ion over another in both the second metal-binding site and the high-affinity sites, we conducted a series of detailed molecular simulations on the EcNikR structure. Simulations that place $\mathrm{Ni}^{2+}$ at high-affinity sites lead to stable trajectories with realistic ion-ligand distances and geometries, while simulations that place $\mathrm{K}^{+}$at these sites lead to conformational changes in the protein that are likely unfavorable for ion binding. By contrast, simulations on the second metal site in the EcNikR-DNA complex lead to stable trajectories with realistic geometries regardless of whether $\mathrm{K}^{+}$or $\mathrm{Ni}^{2+}$ occupies this site. Electrostatic binding free energy calculations, however, suggest that $E c$ NikR binding to DNA is more favorable when the second metal-binding site contains $\mathrm{K}^{+}$. An analysis of the energetic contributions to the electrostatic binding free energy suggests that, while the interaction between $E c$ NikR and DNA is more favorable when the second site contains $\mathrm{Ni}^{2+}$, the large desolvation penalty associated with moving $\mathrm{Ni}^{2+}$ from solution to the relatively buried second site offsets this favorable interaction term. Additional free energy simulations that account for both electrostatic and non-electrostatic effects argue that $E c$ NikR binding to DNA is most favorable when the second site contains a monovalent ion the size of $\mathrm{K}^{+}$. Taken together, these data suggest that the $E c$ NikR structure is

\footnotetext{
(C) 2009 American Chemical Society

cmstultz@csail.mit.edu.

†Department of Chemistry.

\$Department of Physics.

$\S$ Department of Biology.

Department of Electrical Engineering and Computer Science.

$\perp_{\text {Research Laboratory of Electronics. }}$

\# Howard Hughes Medical Institute.

$\nabla_{\text {Harvard-MIT Division of Health Sciences and Technology. }}$
}

Supporting Information Available: Average energies for both the $E c$ NikR high-affinity site studies (Figure S1) and the EcNikR-DNA second metal-binding site studies (Figure S3); metal-ligand distances at one of the four high-affinity metal-binding sites for the four known ligands during the EcNikR high-affinity site studies (Figure S2). This material is available free of charge via the Internet at http://pubs.acs.org. 
most stable when $\mathrm{Ni}^{2+}$ occupies high-affinity sites and that $E c$ NikR binding to DNA is more favorable when the second site contains $\mathrm{K}^{+}$.

\section{Introduction}

Monitoring the concentration of metal ions within the cell is of crucial importance, as a relatively small concentration of some metal ions is necessary for a number of cellular processes to occur, but often too much of that same ion may cause cell damage and death. ${ }^{1-4}$ Nickel is an essential cofactor for a number of bacterial enzymes that are involved in cell survival, including hydrogenase and urease, two enzymes vital to the survival of the pathogenic bacteria Helicobacter pylori. ${ }^{1}$ The concentration of nickel in many bacteria is regulated by the nickel-binding transcription factor, NikR, which transcriptionally represses genes encoding nickel-specific importers. ${ }^{5}$

The best-characterized NikR homologues are from Escherichia coli (EcNikR), H. pylori ( $H p \mathrm{NikR})$, and Pyrococcus horikoshi (PhNikR) ${ }^{6-9}$ Each is a homotetramer composed of two domains: the central tetrameric metal-binding domain (MBD) and two flanking dimeric ribbon-helix-helix (RHH) domains (Figure 1a). The MBD is composed of C-terminal residues 48-133 (E. coli numbering), and each monomer contains a single high-affinity square-planar nickel-binding site composed of H87, H89, and C95 from one monomer and H76 from a neighboring monomer (Figure 1a). The RHH DNA-binding portion of the protein is composed of residues $1-47$ and is arranged in a common DNA-binding motif such that the $\mathrm{N}$-terminal ribbons from two monomers create a two-stranded $\beta$ sheet that inserts into the major groove of DNA. The RHH domains are separated from the MBD by flexible linkers, and crystal structures have captured the RHH in a number of different arrangements with respect to the MBD, suggesting that the RHH domains are free to move with respect to the MBD. ${ }^{8,9}$ However, the relevant DNA-binding conformation for the RHH domains appears to be a "down-cis" arrangement, as seen in the EcNikR-DNA crystal structure (Figure 1b). ${ }^{9}$ In addition, two identical second metal-binding sites are formed when NikR assumes a "down-cis" arrangement and are located at the interface of the RHH and MBD domains (Figure 1b). These second metal-binding sites are composed of two conserved amino acid residues from the RHH domain, E30 and D34, and a number of backbone carbonyls from residues in the MBD (Figure 1b). ${ }^{9}$

Biochemical data and crystal structures of various NikR proteins have informed as well as raised interesting questions regarding these metal-binding sites. The high-affinity metalbinding sites have been well characterized as nickel-binding sites, which upon nickel binding induce a conformational change allowing NikR to bind DNA. ${ }^{10,11}$ Interestingly, nickel binding does not appear to affect the conformation of the RHH DNA-binding domains that make specific contacts to DNA, instead altering the structure of the MBD that makes nonspecific DNA contacts (Figure 1). ${ }^{9}$ In particular, $\mathrm{Ni}^{2+}$ binding orders the a-helix 3 through its coordination of helical residue H76; conversely, $\mathrm{H} 76$ and a-helix 3 are disordered in the apo EcNikR structure. ${ }^{8}$

Crystallographic and mutagenesis data suggest that, like NikR's high-affinity site, the second metal-binding sites are also important in modulating the affinity of NikR for DNA. ${ }^{9}$ The location of these sites at the interface of the MBD and RHH domains suggests that a metal in this site would stabilize the "down-cis" or the DNA-binding mode of $E c$ NikR. Although there has been general agreement that these sites are functionally important, opinions have varied about which metal is likely to occupy them in vivo, with the contenders being $\mathrm{Ni}^{2+}$ and $\mathrm{K}^{+} .6,9$ The suggestion that $\mathrm{Ni}^{2+}$ occupies these second sites comes from biochemical as well as crystallographic data. In particular, metal-binding studies have shown that the affinity of $E c N i k R$ for DNA increases from nanomolar, when 
stoichiometric $\mathrm{Ni}^{2+}$ ions are bound to the high-affinity nickel-binding sites, to picomolar, when excess nickel is present. ${ }^{11,12}$ This increased DNA-binding affinity with excess nickel suggests the presence of one or multiple low-affinity $\mathrm{Ni}^{2+}$-binding sites on $E c \mathrm{NikR}$. The idea that one of these low-affinity sites could be the second metal site is supported by crystallographic data on PhNikR. ${ }^{6,13}$ Here, however, the high concentration of $\mathrm{Ni}^{2+}$ used (20 $\mathrm{mM}$ ) and the need for phosphate in the crystallization conditions raises questions about the physiological relevance of these structural data. In addition, crystallographic data on $E c$ NikR do not support the idea that $\mathrm{Ni}^{2+}$ binds in the second metal sites, instead showing multiple low-affinity sites on the surface of the EcNikR protein. ${ }^{9}$

The suggestion that $\mathrm{K}^{+}$might be the relevant metal in the second metal-binding sites also comes from both biochemical and structural data. Sheila Wang and Deborah Zamble have recently shown that $\mathrm{K}^{+}$is required for $E c$ NikR-DNA binding under both stoichiometric and excess nickel conditions (personal communication). The localization of this $\mathrm{K}^{+}$effect to the second metal-binding sites is derived from the structure of the EcNikR-DNA complex, in which a $\mathrm{K}^{+}$ion from the crystallization buffer is modeled in this site. ${ }^{9}$ Although the binding of ions from the crystallization buffer is often an artifact, in this case the concentration of $\mathrm{K}^{+}$ in the crystallization buffer $(200 \mathrm{mM})$ is slightly lower than that in E. coli $(250 \mathrm{mM})$, indicating that the presence of $\mathrm{K}^{+}$in the second metal-binding sites of this structure could be physiologically relevant. ${ }^{9}, 14$ If these second metal-binding sites are $\mathrm{K}^{+}$sites, then they would not be regulatory sites but rather structural sites in which the binding of abundant cellular monovalent ions affords additional stability to the DNA-bound conformation of EcNikR.

To decipher the physical basis underlying any difference in metal-binding specificity at both the high-affinity and second metal-binding sites, we conducted a series of detailed molecular simulations on the $E c$ NikR structure. We begin with an analysis of the $E c N i k R$ structure when either $\mathrm{K}^{+}$or $\mathrm{Ni}^{2+}$ occupies the high-affinity or the second metal-binding sites. We then explore the effect of the identity of the ion in the second metal-binding site on EcNikR-DNA binding using a combined molecular mechanics Poisson-Boltzmann (MMPB) approach. ${ }^{15}$ Finally, we use detailed free energy simulations to explore the effect ion size in the second metal-binding site has on EcNikR-DNA association. In total, these data clarify the physical basis for metal ion specificity in both the high-affinity and second metal-binding sites in EcNikR.

\section{Results}

\section{Conformation of High-Affinity Sites in the Presence of $\mathrm{Ni}^{2+}$ or $\mathrm{K}^{+}$}

The high-affinity metal-binding sites on $E c N i k R$ have been well-characterized as squareplanar $\mathrm{Ni}^{2+}$-binding sites (Figure 1a). ${ }^{8,16,17}$ While this site is preferentially a $\mathrm{Ni}^{2+}$-binding site, in vitro metal-binding studies have shown that the coordinating species are flexible and can rearrange under different conditions. ${ }^{8,17,18}$ In light of this, we explored whether highaffinity sites in $E c$ NikR could rearrange to accommodate $\mathrm{K}^{+}$, which is present at much higher concentrations in the intracellular space than $\mathrm{Ni}^{2+}$. Starting our studies with the highaffinity sites allows us to explore the preference for $\mathrm{Ni}^{2+}$ versus $\mathrm{K}^{+}$under conditions where the identity of the physiologically relevant metal is unquestioned.

Extensive energy minimizations of the $\mathrm{Ni}^{2+}$-containing high-affinity sites do not alter the geometry or the ion-ligand distances relative to those in the crystal structure (Figure 2a). To determine potential binding modes for $\mathrm{K}^{+}$in the high-affinity sites, we replaced $\mathrm{Ni}^{2+}$ with $\mathrm{K}^{+}$and energy-minimized the system. The objective of these calculations was to determine if there are flexible moieties in the vicinity of the high-affinity site that could participate in metal coordination, thereby yielding a geometry that was favorable for $\mathrm{K}^{+}$. However, the 
energy-minimized structure does not significantly deviate from a square-planar geometry (Figure 2b). While this geometry is expected for $\mathrm{Ni}^{2+}$, it is unprecedented for $\mathrm{K}^{+}$. A recent review of metal-ligand geometries of 4277 small-molecule crystal structures and 113 proteins structures suggests that the minimum coordination number for $\mathrm{K}^{+}$is 5 , with 6 being the most common number of coordinating ligands. ${ }^{19}$ In addition, the resulting coordinating species in this site consist of nitrogen and sulfur atoms, known $\mathrm{Ni}^{2+}$ ligands, but less common $\mathrm{K}^{+}$ligands. ${ }^{19,20} \mathrm{~A}$ search of the Cambridge Crystallographic Structural Database indicates that the average $\mathrm{K}^{+}-\mathrm{N}$ distances is $2.9 \AA$ (from 3517 structures) and the average $\mathrm{K}^{+}-\mathrm{S}$ distance is $3.3 \AA$ (from 297 structures), longer than those we see in our energyminimized structure with $\mathrm{K}^{+}$at the high-affinity sites (Figure 2b).

Since typical energy minimization routines find local energy minima in the vicinity of the starting structure, we performed molecular dynamics (MD) simulations of $E c \mathrm{NikR}$ with $\mathrm{K}^{+}$ in the high-affinity sites to sample low-energy states over a larger region of conformational space. The goal was to determine whether the protein can adopt conformations that are consistent with our current understanding of favorable $\mathrm{K}^{+}$-binding geometries. Simulations of $E c \mathrm{NikR}$ with $\mathrm{K}^{+}$in the high-affinity sites were performed for $3.1 \mathrm{~ns}$ in explicit solvent. The average structure arising from these simulations has $\mathrm{K}^{+}$coordinated by six ligands arranged in an octahedral geometry (Figure $2 \mathrm{c}$ ), consistent with prior analyses of $\mathrm{K}^{+}$-binding sites. ${ }^{19}$ In the average structure, three of the original high-affinity site ligands maintain their interactions with the ion with reasonable $\mathrm{K}^{+}$-ligand distances, while the $\mathrm{H} 76$ side chain is displaced by $3.7 \AA$ and the backbone carbonyl of V88, along with two water molecules, aids in coordinating the $\mathrm{K}^{+}$ion (Figure $2 \mathrm{c}$ ). In short, in order for $\mathrm{K}^{+}$to adopt its expected octahedral geometry, the protein "opens" to allow water molecules to enter the high-affinity site during the simulation. For comparison, we also conducted simulations in explicit solvent of $E c \mathrm{NikR}$ with $\mathrm{Ni}^{2+}$ in the high-affinity sites. After more than $3 \mathrm{~ns}$, however, no water penetrates the high-affinity site when it is occupied by $\mathrm{Ni}^{2+}$, and the $\mathrm{Ni}^{2+}$ maintains its interactions with all four coordinating species as shown in Figure 2a.

These data suggest that the high-affinity sites in the protein cannot rearrange to form ion coordination numbers that are consistent with favorable $\mathrm{K}^{+}$-binding geometries. An octahedral geometry is obtained only when additional solvent molecules enter the site to participate in ligand coordination. Moreover, in all NikR structures where the high-affinity $\mathrm{Ni}^{2+}$ ion is appropriately coordinated by four ligands, this site is buried and not solvent exposed. ${ }^{6,8,9}$ In total, these observations suggest that $\mathrm{K}^{+}$-containing high-affinity sites are associated with large conformational changes that cause the protein to significantly deviate from the prototypical NikR structure.

\section{Conformation of the Second Metal-Binding Site in the Presence of $\mathrm{Ni}^{2+}$ or $\mathrm{K}^{+}$}

In the crystal structure of $E c$ NikR bound to DNA, ligands that coordinate the ion in the second metal-binding site are arranged in an octahedral geometry (Figure 1b), and energy minimization of this site does not significantly alter this geometry (Figure 3a). To explore whether the second metal-binding site can rearrange to adopt conformations that are favorable for $\mathrm{Ni}^{2+}$, we replaced $\mathrm{K}^{+}$with $\mathrm{Ni}^{2+}$ in the crystal structure of $E c \mathrm{NikR}$ bound to DNA. Subsequent energy minimization resulted in a distorted square-pyramidal geometry, with a coordination number of 5, and shorter metal-ligand distances (Figure 3b). The shorter metal-ligand distances in the complex with $\mathrm{Ni}^{2+}$ bound at the second metal-binding site are consistent with $\mathrm{Ni}^{2+}$ having a smaller van der Waals radius than $\mathrm{K}^{+}$; studies of protein structures in the Protein Data Bank suggest that the typical $\mathrm{Ni}^{2+}-\mathrm{O}$ distance is $2.2 \AA$, while the average $\mathrm{K}^{+}-\mathrm{O}$ distance is $2.8 \AA .{ }^{19,20}$ In our energy-minimized structures, we also find that the average $\mathrm{Ni}^{2+}-\mathrm{O}$ and $\mathrm{K}^{+}-\mathrm{O}$ distances are 2.2 and $2.8 \AA$, respectively (Figure 3). An analysis of $\mathrm{Ni}^{2+}$ coordination geometries obtained from eight structures in the Protein Data 
Bank and over 1000 structures in the Cambridge Structural Database reveals that similar geometries have been observed in other crystal structures that contain bound $\mathrm{Ni}^{2+}$ ions. ${ }^{20-22}$

Molecular dynamics simulations of $E c \mathrm{NikR}$ complexed to DNA with either $\mathrm{K}^{+}$or $\mathrm{Ni}^{2+}$ in the second metal-binding site confirm that the octahedral geometry and the distorted squarepyramidal geometry for $\mathrm{K}^{+}$and $\mathrm{Ni}^{2+}$, respectively, represent stable geometries for both ions. Over the course of a $2.1 \mathrm{~ns}$ simulation, both metals maintained the previously described binding geometries with reasonable metal-ligand distances. Hence, unlike the previous simulations that uncovered significant structural changes in high-affinity sites when the $\mathrm{Ni}^{2+}$ was replaced with $\mathrm{K}^{+}$, these data suggest that the second metal-binding site can accommodate either ion and substitution with $\mathrm{Ni}^{2+}$ does not lead to significant deviations in the $E c$ NikR-DNA structure.

\section{EcNikR-DNA Association with Either Ni2+ or $\mathrm{K}+$ in the Second Metal-Binding Site}

In light of these data, we explored the effect that $\mathrm{K}^{+}$or $\mathrm{Ni}^{2+}$ binding at the second metalbinding site has on EcNikR-DNA association. We are interested in calculating the difference between the energy associated with $E c$ NikR binding to DNA with $\mathrm{K}^{+}$in the second site $\left(\Delta G_{\mathrm{K}}\right)$ and DNA binding with $\mathrm{Ni}^{2+}$ in the second site $\left(\Delta G_{\mathrm{Ni}}\right)$ (Figure 4$)$. The difference between the two binding energies is equal to the free energy difference between two states; one state has $\mathrm{K}^{+}$in the second site, and the other state has $\mathrm{Ni}^{2+}$ in the second site, and $\Delta \Delta G$ $=\Delta G_{\mathrm{Ni}}-\Delta G_{\mathrm{K}}$. The thermodynamic cycle used for the MMPB calculations is outlined in Figure 4. If $\Delta \Delta G$ is positive, $E c N i k R-D N A$ binding is preferred when $\mathrm{K}^{+}$is in the second metal-binding site, and if $\Delta \Delta G$ is negative, $E c N$ ikR-DNA binding is preferred when $\mathrm{Ni}^{2+}$ is bound.

We note that the individual binding free energies $\left(\Delta G_{\mathrm{K}}\right.$ and $\left.\Delta G_{\mathrm{Ni}}\right)$ can be decomposed into a sum of electrostatic and non-electrostatic contributions: ${ }^{23,24}$

$$
\begin{gathered}
\Delta G_{\mathrm{K}}=\Delta G_{\mathrm{K}}^{\mathrm{elec}}+\Delta G_{\mathrm{K}}^{\mathrm{non}-\mathrm{elec}} \\
\Delta G_{\mathrm{Ni}}=\Delta G_{\mathrm{Ni}}^{\text {elec }}+\Delta G_{\mathrm{Ni}}^{\text {non-elec }}
\end{gathered}
$$

In each case, the non-electrostatic contributions can be expressed as a sum of a hydrophobic term plus additional terms that account for other contributions such as configuration entropy. ${ }^{23-25}$ These non-electrostatic terms are typically estimated from an analysis of the unbound state and the bound complex; e.g., the hydrophobic term is calculated using the buried surface area of the complex. ${ }^{23-25}$ We assume that the EcNikR-binding mode is the same regardless of whether $\mathrm{Ni}^{2+}$ or $\mathrm{K}^{+}$is present in the second metal-binding site, i.e., that the structural changes at the second metal-binding site are small (Figure 3). Consequently, $\Delta G_{\mathrm{Ni}}^{\text {non-elec }} \approx \Delta G_{\mathrm{K}}^{\text {non-elec }}$, and hence $\Delta \Delta G \approx \Delta G_{\mathrm{Ni}}^{\text {elec }}-\Delta G_{\mathrm{K}}^{\text {elec }}$. We therefore use a combined MMPB approach to estimate the overall difference in binding free energy. ${ }^{15}$

We further express $\Delta \Delta G$ as a sum of physically meaningful terms, as outlined in Figure 5, in a manner similar to what has been done in previous studies. ${ }^{23,24,26,27}$ First, the $\mathrm{K}^{+}$-bound $E c$ NikR-DNA complex and two free $\mathrm{Ni}^{2+}$ ions are desolvated $(\mathbf{1} \rightarrow \mathbf{2}$ in Figure 5). Next, in a vacuum, $\mathrm{K}^{+}$ions are exchanged for $\mathrm{Ni}^{2+}$ ions at the second metal-binding sites in the $E c$ NikR-DNA complex with the associated free energy difference of $\Delta G_{\mathrm{e}}(\mathbf{2} \rightarrow \mathbf{3})$.

However, the resulting structure has residues in the second metal-binding sites arranged in an orientation that is optimal for $\mathrm{K}^{+}$, not $\mathrm{Ni}^{2+}$. Therefore, we computed the electrostatic energy associated with residues at the second metal-binding sites rearranging to adopt a 
conformation that is favorable for $\mathrm{Ni}^{2+}$ binding $(\mathbf{3} \rightarrow \mathbf{4})$. In the last step of the thermodynamic path, we calculated the energy associated with solvating the relaxed $\mathrm{Ni}^{2+}$ bound $E c$ NikR-DNA complex and two free $\mathrm{K}^{+}$ions $(\mathbf{4} \rightarrow \mathbf{5})$. Each contribution is calculated from a set of 10 structures taken from a $1 \mathrm{~ns}$ MD simulation in explicit solvent. Values for the various contributions to $\Delta \Delta G$ are shown in Table 1 and represent the average value obtained from the 10 structures arising from the MD simulations.

The calculated value for $\Delta \Delta G$ is large, an average of $733 \mathrm{kcal} \mathrm{mol}^{-1}$ site $^{-1}$, suggesting that $E c$ NikR-DNA binding is preferred when $\mathrm{K}^{+}$occupies the secondary site. To put this number into context, we note that the experimentally determined value for $\mathrm{Ni}^{2+}$ desolvation is also quite large, at $477 \mathrm{kcal} / \mathrm{mol}$ (and similar to the calculated value of $456 \mathrm{kcal} / \mathrm{mol}$ ). ${ }^{28}$

Interestingly, the value of the exchange term, $\Delta G_{\mathrm{e}}$, suggests that replacing $\mathrm{K}^{+}$with $\mathrm{Ni}^{2+}$ is favorable; i.e., $\mathrm{Ni}^{2+}$ makes more favorable interactions with the protein and DNA relative to $\mathrm{K}^{+}$(Table 1). However, this favorable contribution is largely offset by the unfavorable $\mathrm{Ni}^{2+}$ desolvation energy. The other major unfavorable contribution arises from the sum of the desolvation and solvation contributions for the protein DNA complex,

$\Delta G_{\mathrm{d}}^{\mathrm{C}-\mathrm{K}}+\Delta G_{\mathrm{s}}^{\mathrm{C}-\mathrm{Ni}}=\Delta G_{\mathrm{s}}^{\mathrm{C}-\mathrm{Ni}}-\Delta G_{\mathrm{s}}^{\mathrm{C}-\mathrm{K}}=932 \mathrm{kcal} \mathrm{mol}^{-1}$ site $^{-1}$ (where $\Delta G_{\mathrm{s}}^{\mathrm{C}-\mathrm{K}}$ is the electrostatic energy associated with solvating the DNA-bound complex that has $\mathrm{K}^{+}$in the second metalbinding site). The large positive value of this term suggests that solvating the DNA-bound complex is more favorable when $\mathrm{K}^{+}$is in the second metal-binding site. Of note, the complex of $E c \mathrm{NikR}$, DNA, and four high-affinity $\mathrm{Ni}^{2+}$ ions has a very large overall charge of -84 . Addition of two $\mathrm{K}^{+}$ions in the second metal-binding sites brings the total charge to -82 , while the addition of two $\mathrm{Ni}^{2+}$ ions brings the total charge to -80 . As the total charge of the system containing $\mathrm{K}^{+}$is more negative, it is not surprising that it has a larger negative (i.e., more favorable) solvation energy relative to the complex with $\mathrm{Ni}^{2+}$ bound.

The Poisson-Boltzmann formalism allows us to calculate residue-specific contributions to the overall difference in free energy $(\Delta \Delta G)$. Figure 6 highlights the residues with the greatest contribution, positive (blue) or negative (red), to the overall difference in free energy. It is noteworthy that residues that are distant from the secondary site make significant contributions to the overall free energy change. That is, the relative preference for $\mathrm{K}^{+}$in the secondary site cannot be explained using an analysis of the structure of the protein in the vicinity of the second metal-binding site alone.

\section{Effect of lon Size on DNA Binding}

The electrostatic calculations suggest that $E c$ NikR-DNA association is more favorable when $\mathrm{K}^{+}$, and not the divalent ion $\mathrm{Ni}^{2+}$, occupies the second metal-binding site. This large difference is due, in part, to the fact that $\mathrm{Ni}^{2+}$ has a relatively large desolvation penalty. This raises the possibility that $E c$ NikR DNA binding may be afforded by another monovalent cation in the second metal-binding site. In particular, we are interested in how the affinity of $E c$ NikR for DNA changes when ions of different sizes occupy the second metal-binding site. For these studies we focus on $\mathrm{Na}^{+}$, which is considerably smaller than $\mathrm{K}^{+}$, and $\mathrm{Cs}^{+}$, which is larger than $\mathrm{K}^{+}$.

The thermodynamic cycle for these calculations is shown in Figure 7. We are interested in the difference between the binding free energies of two binding reactions. In the first reaction, $E c$ NikR binds DNA with $\mathrm{K}^{+}$in the second metal-binding site, and the associated free energy difference is $\Delta G_{\mathrm{K}}$. In the second reaction, $E c N i k R$ binds DNA with a monovalent ion denoted by $\mathrm{X}^{+}$, and again the associated free energy difference is given by $\Delta G_{\mathrm{X}}$. The relative binding free energy is $\Delta \Delta G_{\mathrm{K} \rightarrow \mathrm{X}} \equiv \Delta G_{\mathrm{X}}-\Delta G_{\mathrm{K}}=\Delta G_{\mathrm{K} \rightarrow \mathrm{X}}^{\mathrm{P}}-\Delta G_{\mathrm{K} \rightarrow \mathrm{X}}^{\mathrm{S}}$. The overall $\Delta \Delta G$ can therefore be calculated using the contributions along the alchemical paths, $\Delta G_{\mathrm{K} \rightarrow \mathrm{X}}^{\mathrm{P}}, \Delta G_{\mathrm{K} \rightarrow \mathrm{X}}^{\mathrm{S}}$. 
Ions can be characterized by a distinct van der Waals radius, $\sigma$, which determines the equilibrium interatomic distance, and the well-depth, $\varepsilon$. In Figure $7, \mathrm{X}^{+}$represents a monovalent ion having a van der Waals radius and well-depth different from those of $\mathrm{K}^{+}$.

Therefore, we calculate $\Delta G_{\mathrm{K} \rightarrow \mathrm{X}}^{\mathrm{P}}$ and $\Delta G_{\mathrm{K} \rightarrow \mathrm{X}}^{\mathrm{S}}$ in a stepwise fashion, where first we calculate the effect of changing $\sigma$ and then we compute the effect of changing the well-depth, $\boldsymbol{\varepsilon}$. For example, we express the free energy differences along the alchemical paths associated with transforming $\mathrm{K}^{+}$to $\mathrm{Na}^{+}$as

$$
\begin{aligned}
& \Delta G_{\mathrm{K} \rightarrow \mathrm{Na}}^{\mathrm{S}}=\Delta G_{\mathrm{K}\left(\sigma_{\mathrm{K}}+\varepsilon_{\mathrm{K}^{+}}\right) \rightarrow Y\left(\sigma_{\mathrm{Na}^{+}}+, \varepsilon_{\mathrm{K}}^{+}\right)}^{\mathrm{S}}+\Delta G_{\mathrm{Y}\left(\sigma_{\mathrm{Na}}+, \varepsilon_{\mathrm{K}}+\right) \rightarrow \mathrm{Na}\left(\sigma_{\mathrm{Na}}+, \varepsilon_{\mathrm{Na}}+\right)}^{\mathrm{S}} \\
& \Delta G_{\mathrm{K} \rightarrow \mathrm{Na}}^{\mathrm{P}}=\Delta G_{\mathrm{K}\left(\sigma_{\mathrm{K}}+\varepsilon_{\mathrm{K}^{+}}\right) \rightarrow Y\left(\sigma_{\mathrm{Na}^{+}}+, \varepsilon_{\mathrm{K}}^{+}\right)}^{\mathrm{P}}+\Delta G_{\mathrm{Y}\left(\sigma_{\mathrm{Na}}+, \varepsilon_{\mathrm{K}}+\right) \rightarrow \mathrm{Na}\left(\sigma_{\mathrm{Na}}+, \varepsilon_{\mathrm{Na}}+\right)}^{\mathrm{P}}
\end{aligned}
$$

While the individual contributions to the total free energy change are not state functions, the total free energy change is. ${ }^{29}$ Therefore, the precise path chosen to calculate the overall free energy will not, in principle, affect the final result. Each term on the right-hand side of eqs 3 and 4 is computed using thermodynamic integration with a linear alchemical path. The overall free energy differences are listed in Table 2. For both $\mathrm{Na}^{+}$and $\mathrm{Cs}^{+}$, the relative binding affinities are positive, suggesting that DNA binding is more favorable when the second site contains $\mathrm{K}^{+}$.

\section{Discussion}

The metal-binding sites of the transcriptional repressor $E c$ NikR have been the subject of much attention. ${ }^{8-11,16-18,30}$ While the role of the high-affinity $\mathrm{Ni}^{2+}$-binding site as the main regulator of EcNikR's affinity for DNA has not been in question, the function of the second metal site has been less clear. Possibilities include that this second site is an additional regulatory site to which excess $\mathrm{Ni}^{2+}$ ions can bind, yielding a tighter $E c$ NikR-DNA complex. Alternatively, this second site could be non-regulatory, binding the abundant cellular $\mathrm{K}^{+}$ion to further stabilize the $E c$ NikR-DNA interaction. Here we consider whether both $\mathrm{K}^{+}$and $\mathrm{Ni}^{2+}$ can stably bind this second site. Using unconstrained MD simulations of the $E c$ NikR-DNA complex with either $\mathrm{K}^{+}$or $\mathrm{Ni}^{2+}$ in the second metal site, we show that either of these metals is a reasonably good fit for the site. The overall backbone average root-mean-square deviations of the EcNikR-DNA complex with either metal bound at the second metal site are very similar, and in both cases, the second metal-binding site adopts realistic ion-ligand geometries. In light of these observations, we explored the effect of having either $\mathrm{Ni}^{2+}$ or $\mathrm{K}^{+}$in the second metal-binding site on $E c$ NikR-DNA association. Electrostatic free energy calculations suggest that, while the Coulombic interactions between $\mathrm{Ni}^{2+}$ and the $E c$ NikR-DNA complex are more favorable relative to those with $\mathrm{K}^{+}$, this favorable contribution is counterbalanced by the unfavorable terms associated with the desolvation penalty of $\mathrm{Ni}^{2+}$ and the EcNikR-DNA complex. An analysis of the individual residue contributions to the overall binding free energies suggest that the preference for $\mathrm{K}^{+}$ at the second metal-binding site cannot be explained from an analysis of the local conformation of the protein in the vicinity of the second metal-binding site. Residues that are distant from the second metal-binding site have a significant effect on the identity of the ion in this site (Figure 6). These data raise the interesting possibility that mutations at sites that are distant from the second metal-binding site can affect the ion that is present in the bound complex.

As the high desolvation penalty of $\mathrm{Ni}^{2+}$ is partially responsible for the $\mathrm{K}^{+}$being preferred in the second metal-binding site, EcNikR-DNA binding may be possible with any monovalent 
ion in the second metal-binding site, since these ions have much smaller desolvation penalties. ${ }^{28,31}$ Additional free energy simulations that examine the effect of having other monovalent ions at the secondary site on EcNikR-DNA association suggest that the affinity of $E c$ NikR for DNA is significantly reduced when the second metal-binding site contains $\mathrm{Na}^{+}$, a common monovalent cation in the cellular milieu (Table 2).

With the finding that desolvation is the main source of preference for $\mathrm{K}^{+}$over $\mathrm{Ni}^{2+}$ in the second metal site, it is important to understand why the high-affinity site binds $\mathrm{Ni}^{2+}$ and not $\mathrm{K}^{+}$, especially given the high concentration of $\mathrm{K}^{+}$compared to $\mathrm{Ni}^{2+}$ in E. coli. We found that the second metal site can rearrange to stably bind both metals, raising the question of whether the same is true for the high-affinity sites. Replacing $\mathrm{Ni}^{2+}$ with $\mathrm{K}^{+}$at high-affinity sites, followed by energy minimization, however, does not lead to ion-ligand geometries that are consistent with what one would expect for $\mathrm{K}^{+}$. Energy-minimized high-affinity sites that contain $\mathrm{K}^{+}$do not form an octahedral geometry, the preferred binding geometry for $\mathrm{K}^{+} .{ }^{19}$ Moreover, the coordination number for $\mathrm{K}^{+}$at these sites is 4 , whereas the minimum coordination number for $\mathrm{K}^{+}$in both small-molecular and protein crystal structures is $5 .{ }^{19}$ Additional MD simulations of $E c$ NikR with $\mathrm{K}^{+}$at high-affinity sites suggest that realistic coordination geometries for $\mathrm{K}^{+}$cannot be achieved with moieties that arise solely from the protein. That is, an octahedral geometry is achieved only when the protein undergoes significant conformational changes that cause the high-affinity sites to become solvent exposed. These data suggest that the $E c \mathrm{NikR}$ structure cannot accommodate $\mathrm{K}^{+}$at the highaffinity sites.

Additional insights into the effect of $\mathrm{K}^{+}$on the $E c \mathrm{NikR}$ structure are obtained from an analysis of its coordinating species in the MD simulations. After $3 \mathrm{~ns}$ of molecular dynamics, $\mathrm{K}^{+}$achieves an octahedral geometry where two of the ligands are water molecules. The side chain of $\mathrm{H} 76$, which normally participates in ion coordination, shifts to a position where it can no longer coordinate the ion in the site (Figures 2c and S2 in the Supporting Information). This observation is of interest because structural studies have linked ion ligation at high-affinity sites by residue $\mathrm{H} 76$ with ordering of the a 3 helix in the MBD, which contains residue H76. ${ }^{9,18}$ The ordering of the a 3 helix upon $\mathrm{Ni}^{2+}$ binding has been implicated in the mechanism of EcNikR-DNA binding, and solution studies suggest that metals that are capable of ordering the a 3 helix are those that induce $E c$ NikR-DNA binding. ${ }^{9}, 11,12,18,30$ Metals that do use $\mathrm{H} 76$ as a ligand, such as $\mathrm{Ni}^{2+}$ and $\mathrm{Cu}^{2+}$, have wellordered a 3 helices. ${ }^{8,18}$ However, when no metal or a metal like $\mathrm{Zn}^{2+}$ that does not induce strong DNA binding is bound at the high-affinity site, H76 is not utilized as a ligand, and the a3 helix is relatively disordered. ${ }^{18}$ Although the time scale of our simulations is not sufficiently long to reliably examine helix stability in the presence of $\mathrm{K}^{+}$, these data suggest that $\mathrm{K}^{+}$does not induce the proper ligand ordering at the high-affinity site and may not support a 3 helix stability or, in turn, proper DNA binding.

While classical dynamical simulations and continuum electrostatic calculations have a long history of providing useful insights into a number of biological problems, it is important to note that typically a number of approximations are made to yield results in a reasonable amount of CPU time. ${ }^{33,34}$ In particular, our calculations account for only a relatively small region of DNA that is known to bind $E c N i k R$ and that was present in the $E c$ NikR co-crystal structure. ${ }^{9}$ As DNA is highly charged and electrostatic interactions are long range in character, distant regions of DNA may influence the relative affinity of different ions for the second metal-binding site in vivo. Indeed, our results suggest that relatively distant residues on the EcNikR protein itself may influence the identity of the ion in the second metalbinding site. 
Nevertheless, our data explain why $\mathrm{Ni}^{2+}$ is preferred over $\mathrm{K}^{+}$at high-affinity sites and suggest that $E c$ NikR binding to DNA is more favorable when $\mathrm{K}^{+}$is present at the second metal-binding site. Recent studies have explored the effect of $\mathrm{K}^{+}$on $E c$ NikR-DNA binding using DNase footprinting and gel mobility shift assays. These results are consistent with our findings in that they demonstrate that $\mathrm{K}^{+}$is required for $E c$ NikR-DNA association in vitro (Sheila Wang and Deborah Zamble, personal communication).

In conclusion, $E c \mathrm{NikR}$ is exquisitely designed with a number of metal-binding sites of varying metal preferences. Other NikR homologues, such as $H p N i k R$, have been shown to also require multiple metal-binding sites, and interestingly some of these sites are believed to be distinct from the sites in $E c$ NikR. ${ }^{35,36}$ Consequently, NikR proteins from different bacteria may have different metal-binding properties, and hence the NikR family represents a diverse and complex set of metal regulatory proteins. The central high-affinity $\mathrm{Ni}^{2+}$ binding sites, which are conserved among all NikR homologues, are sensory sites that specifically bind the scarce cellular $\mathrm{Ni}^{2+}$ ion, enabling binding to the nik promoter. The second metal-binding sites in $E c$ NikR are located at the interface of the MBD and RHH domains and are composed solely of oxygen ligands from each domain. This conserved second metal-binding site is a structural site that prefers the cellularly abundant $\mathrm{K}^{+}$ion to help stabilize the EcNikR-DNA interaction. By utilizing two different metals in each of these sites, EcNikR optimizes its specificity and strength of DNA binding.

\section{Methods}

\section{Structure Preparation and Solvation}

Structures of $\mathrm{Ni}^{2+}$-bound $E c \mathrm{NikR}$ and $E c \mathrm{NikR}-\mathrm{DNA}$ were obtained from the Protein Data Bank (PDB IDs 2HZA and 2HZV, respectively). ${ }^{9,32}$ Missing protein residues were added with only backbone and $\mathrm{C}_{\beta}$ atoms in approximate positions in COOT. ${ }^{37}$ Missing heavy atoms and all hydrogen atoms were built with ICBuild and HBuild functions in CHARMM version 33b2 using the all-atom (CHARMM27) parameters for proteins, nucleic acids, and potassium ions. ${ }^{38}$ Nickel parameters (van der Waals radius of $1.4125 \AA$ and $\mathcal{\varepsilon}$ of -0.010 ) were obtained from Dr. Johan Bredenberg (personal communication) and thiolate parameters from published studies. ${ }^{39}$ Histidines were modeled as $\delta$ - or $\varepsilon$-protonated, depending on the likely hydrogen-bonding pattern; i.e., heavy atoms of hydrogen-bonding residues that were within $3.5 \AA$ of one another suggest that the corresponding nitrogen on His should be protonated. The high-affinity nickel ligand C95 was modeled as a deprotonated thiolate. Following these modifications to the protein-DNA complex, 100 steps of steepest decent minimization were run in a vacuum to remove bad contacts. For all simulations, the SHAKE command was used to restrain bonds involving hydrogen atoms near their equilibrium values, and the nonbonded cutoff was set to $13.0 \AA{ }^{40}$

\section{Minimization and Molecular Dynamics of the High-Affinity Site in EcNikR}

The $E c$ NikR protein was prepared as described above with either four $\mathrm{Ni}^{2+}$ or four $\mathrm{K}^{+}$ions filling the high-affinity metal-binding sites. Simulations with either $\mathrm{Ni}^{2+}$ or $\mathrm{K}^{+}$ions in the high-affinity sites were performed in explicit solvent using a stochastic boundary approach. ${ }^{41}$ The system was solvated using TIP3P water molecules with a sphere of radius $39 \AA$ centered around the metal-binding domain, and a stochastic boundary potential was used to maintain the water sphere around the system. ${ }^{42,43}$ This water sphere allowed for a buffer of $\sim 15 \AA$ between the protein exterior and the boundary of the water sphere. There were a total of 5900 water molecules in the simulation, which were minimized using 500 steps of steepest decent, holding the protein portion fixed. Each of the high-affinity metal sites in both $\mathrm{Ni}^{2+}-E c \mathrm{NikR}$ and $\mathrm{K}^{+}-E c \mathrm{NikR}$ structures were minimized within $5 \AA$ of each 
site using 500 steps of conjugate gradient and holding the rest of the protein and water fixed. Minimizations that employed a larger cutoff did not significantly change our results.

Following the minimization studies, the system was partitioned for the MD simulations in the following way. All solvent molecules and all protein atoms and ions within $39 \AA$ of the center of the protein were in the reaction region and underwent full MD simulation. The protein molecules outside the sphere were constrained by force constants derived from the $B$-factors of the atoms in the X-ray crystal structure. The $B$-factors for atoms that were built into the structure and thus did not have crystallographic $B$-factors were set to the average $B$ factor, depending on that type of atom and its location in the main chain or side chain. $B$ factors were converted to force constants as previously described. ${ }^{41}$

Molecular dynamics simulations were run using CHARMM. ${ }^{38}$ First, the protein was gently heated from 0 to $300 \mathrm{~K}$ at a rate of $5 \mathrm{~K} / \mathrm{ps}$ using a Nosé-Hoover heat bath. ${ }^{44}$ Dynamical simulations then ran at $300 \mathrm{~K}$ until the system had been allowed to fully equilibrate (Figure $\mathrm{S} 1$, Supporting Information). The total run time was $3.1 \mathrm{~ns}$. Structures were saved every 10 ps. From the final $600 \mathrm{ps}$ of the simulation, an average structure was calculated and briefly minimized to remove bad contacts using 100 steps of steepest descent.

\section{Minimization and Molecular Dynamics of the Second Metal-Binding Site in the EcNikR- DNA Complex}

For the studies of the second metal-binding site, the $\mathrm{Ni}^{2+}-E c \mathrm{NikR}-\mathrm{DNA}$ structure was prepared as described above with either $\mathrm{K}^{+}$or $\mathrm{Ni}^{2+}$ in the second metal-binding site and always with $\mathrm{Ni}^{2+}$ at all four high-affinity sites. The protein was solvated using a $57 \AA$ sphere of water (with a total of 21331 water molecules) in order to fully solvate each second metalbinding site with a water buffer of $\sim 15 \AA$. In general, simulations with DNA require explicit solvent and counterions to achieve realistic geometries. ${ }^{42}$ However, the inclusion of specific counterions can lead to relatively long convergence times for systems of modest size. ${ }^{45}$

Therefore, we again employed a stochastic boundary approach where the DNA is restrained using the crystallographically determined $B$-factors and no explicit counterions are used in the dynamic simulations. ${ }^{46}$ This represents a compromise between computational efficiency and accuracy and helps to ensure that the DNA does not sample unrealistic conformations.

Minimization studies of the second metal-binding sites were performed as described for the high-affinity binding sites. For dynamics simulations, the system was partitioned such that all solvent, protein, and ions underwent full dynamics, but the DNA was constrained using harmonic restraints derived from $B$-factors. The same heating, equilibration, and production procedure described above was used (Figure S3, Supporting Information). The total MD run time was $2.1 \mathrm{~ns}$, with the last $600 \mathrm{ps}$ being used to construct the average structure.

\section{MMPB Studies}

For the Poisson-Boltzmann studies we first conducted additional dynamical simulations to obtain representative structures that could be used for electrostatic calculations. In these calculations, the four atoms directly coordinating each nickel ion at the high-affinity metalbinding sites were constrained using an improper dihedral angle of $0^{\circ}$ with force constant of $100 \mathrm{kcal} / \AA^{2}$ to ensure that optimal square-planar $\mathrm{Ni}^{2+}$ coordination geometry was maintained. The second metal sites were minimized as described above with either $\mathrm{K}^{+}$or $\mathrm{Ni}^{2+}$ in the sites. After this minimization, the second metal sites were examined and then the final minimized geometry and distances noted (Figure 3 ). The second metal-binding sites were constrained to the distances defined in Figure 3 using the 'resdistance' command with a force constant of $1.0 \mathrm{kcal} / \AA^{2}$. Similar approaches have been employed in other studies of protein complexes that contain metal-binding sites. ${ }^{47-49}$ 
The protein-DNA complex was solvated in a water sphere of $35 \AA$ centered around the MBD portion of the structure and fully solvating both second metal-binding sites. A total of 3062 water molecules were added, and the system was partitioned and heated as previously described. Given the restraints at the high-affinity and second metal-binding sites, only $80 \mathrm{ps}$ of equilibration was needed, followed by 1 ns of production dynamics. Ten structures were taken from the $1 \mathrm{~ns}$ production trajectory, one every $100 \mathrm{ps}$. At that point the water molecules were deleted, and the 10 representative structures were ready for PoissonBoltzmann calculations.

The program Delphi was used to solve the nonlinear Poisson-Boltzmann equation for each of the complexes. The initial grid size was $250 \AA$ square with a grid spacing of $1 \AA$, which was then focused to a grid size of $125 \AA$ with a grid spacing of $0.5 \AA$. The interior dielectric of the protein was set to 4 for all calculations. For the vacuum calculations, the exterior dielectric was set to 1 , and for calculations in water the exterior dielectric was set to 80 with a salt concentration of $140 \mathrm{mM}$. The energies for all 10 structures in each of the five states along the pathway in Figure 5 were then averaged, and the electrostatic free energy differences were computed.

\section{Thermodynamic Integration}

We calculated the free energy difference between the EcNikR-DNA complex structure with $\mathrm{K}^{+}$bound and the structure with an ion $\mathrm{X}^{+}$, where $\mathrm{X}^{+}$is either $\mathrm{Na}^{+}$or $\mathrm{Cs}^{+}$. The thermodynamic paths of interest are depicted as the horizontal legs of the thermodynamic cycle in Figure 7: $E c N i k R$ binding to DNA with potassium $\left(\Delta G_{\mathrm{K}}\right)$ or to a monovalent ion with a radius larger or smaller than that of potassium $\left(\Delta G_{\mathrm{X}}\right)$. We are interested in the difference of these energies, or $\Delta \Delta G$, where $\Delta \Delta \mathrm{G}_{\mathrm{K} \rightarrow \mathrm{X}} \equiv \Delta G_{\mathrm{X}}-\Delta G_{\mathrm{K}}=\Delta G_{\mathrm{K} \rightarrow \mathrm{X}}^{\mathrm{P}}-\Delta G_{\mathrm{K} \rightarrow \mathrm{X}}^{\mathrm{S}}$. We note that, for an exact calculation of $\Delta G_{\mathrm{K} \rightarrow \mathrm{X}}^{\mathrm{S}}$ and $\Delta G_{\mathrm{K} \rightarrow \mathrm{X}}^{\mathrm{P}}$, we must account for both the radius of the atom, $\sigma$, and the well-depth, $\varepsilon$. From eqs 3 and $4, \Delta G_{\mathrm{K} \rightarrow \mathrm{X}}^{\mathrm{S}}$ and $\Delta G_{\mathrm{K} \rightarrow \mathrm{X}}^{\mathrm{P}}$ can be decomposed into two steps, a $\Delta G_{\mathrm{K} \rightarrow \mathrm{Y}}$ step where only the ionic radius is changed

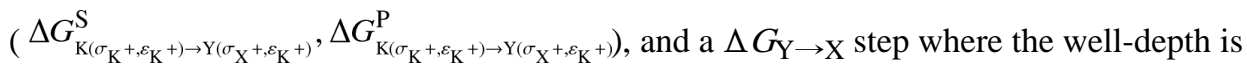

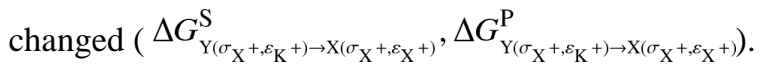

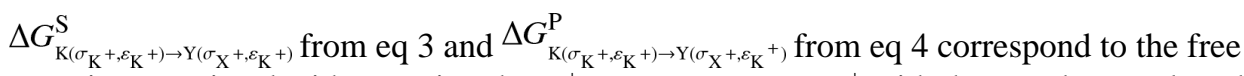
energies associated with mutating the $\mathrm{K}^{+}$atom to an atom $\mathrm{Y}^{+}$with the van der Waals radius of $\mathrm{X}^{+}$but the $\mathcal{E}$ of $\mathrm{K}^{+}(-0.087)$ in solvent or in the EcNikR-DNA complex, respectively. To

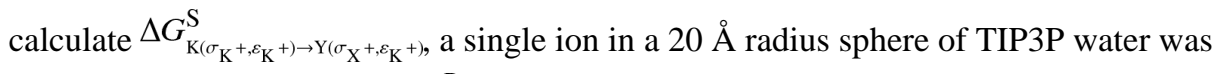

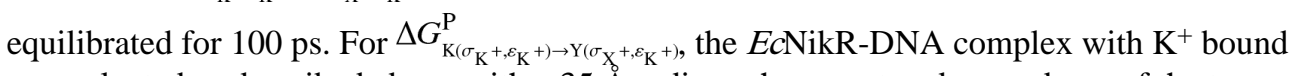
was solvated as described above with a $35 \AA$ radius sphere centered around one of the two second metal-binding sites and equilibrated for 100,200 , or $500 \mathrm{ps}$. These structures were used as starting points for two separate alchemical transformation experiments described below.

The potential energy used for the alchemical transformation is given by $V(\lambda)=V_{\mathrm{K}}+\lambda\left(V_{\mathrm{Y}}\right.$ - $V_{\mathrm{K}}$ ), where $V_{\mathrm{K}}$ and $V_{\mathrm{Y}}$ denote the potential energy of the EcNikR-DNA complex with either $\mathrm{K}^{+}$or an ion $\mathrm{Y}^{+}$bound or the ion free in solution. The free energy difference between the states of EcNikR-DNA with $\mathrm{K}^{+}$or $\mathrm{Y}^{+}$bound is given by

$\Delta G_{\mathrm{K}\left(\sigma_{\mathrm{K}^{+}}, \varepsilon_{\mathrm{K}}+\right) \rightarrow \mathrm{Y}\left(\sigma_{\mathrm{X}^{+}}, \varepsilon_{\mathrm{K}}+\right)}^{\mathrm{P}}=\int_{0}^{1}\langle\Delta V\rangle_{\lambda} \mathrm{d} \lambda$, where $\Delta V=V_{\mathrm{Y}}-V_{\mathrm{K}}$. Simulations were performed for both the solvated $E c$ NikR-DNA complex and the solvated free ions at $\lambda=0.02,0.1,0.2,0.3$, $0.4,0.5,0.6,0.7,0.8,0.9$, and 0.98 . At each $\lambda$ value, $50 \mathrm{ps}$ of equilibration was followed by 
$50 \mathrm{ps}$ of production dynamics. The final free energy differences were computed using a standard quadrature. Electrostatic and non-electrostatic contributions to the free energy change were calculated separately as previously described..$^{50}$

To determine the contribution of the well-depth to the difference in free energy, we ran an experiment similar to that described above, but in this case we calculated the difference in free energy between having an atom $\mathrm{Y}^{+}$with the size of $\mathrm{X}^{+}$and the well-depth of $\mathrm{K}^{+}$, to an atom with both the size and well-depth of $\mathrm{X}^{+}$.

\section{Supplementary Material}

Refer to Web version on PubMed Central for supplementary material.

\section{Acknowledgments}

The authors thank Deborah Zamble and Sheila Wang for helpful discussions.

\section{References}

1. Davis GS, Flannery EL, Mobley HLT. Infect Immun. 2006; 74:6811-6820. [PubMed: 17030579]

2. Mulrooney SB, Hausinger RP. FEMS Microbiol Rev. 2003; 27:239-261. [PubMed: 12829270]

3. Unden G, Bongaerts J. Biochim Biophys Acta. 1997; 1320:217-234. [PubMed: 9230919]

4. Wu LF, Navarro C, de Pina K, Quenard M, Mandrand MA. Environ Health Perspect. 1994; 102(Supplement 3):297-300. [PubMed: 7843119]

5. Dosanjh NS, Michel SLJ. Curr Opin Chem Biol. 2006; 10:123-130. [PubMed: 16504569]

6. Chivers PT, Tahirov TH. J Mol Biol. 2005; 348:597-607. [PubMed: 15826657]

7. Dian C, Schauer K, Kapp U, McSweeney SM, Labigne A, Terradot L. J Mol Biol. 2006; 361:715730. [PubMed: 16872629]

8. Schreiter ER, Sintchak MD, Guo Y, Chivers PT, Sauer RT, Drennan CL. Nat Struct Biol. 2003; 10:794-799. [PubMed: 12970756]

9. Schreiter ER, Wang SC, Zamble DB, Drennan CL. Proc Natl Acad Sci USA. 2006; 103:1367613681. [PubMed: 16945905]

10. Chivers PT, Sauer RT. J Biol Chem. 2000; 275:19735-19741. [PubMed: 10787413]

11. Chivers PT, Sauer RT. Chem Biol. 2002; 9:1141-1148. [PubMed: 12401498]

12. Bloom SL, Zamble DB. Biochemistry. 2004; 43:10029-10038. [PubMed: 15287730]

13. Kitao T, Kuroishi C, Tahirov TH. Acta Crystallogr, Sect F: Struct Biol Cryst Commun. 2005; F61:43-45.

14. Epstein W, Schultz SG. J Gen Physiol. 1966; 49:469-81. [PubMed: 5328217]

15. Kollman PA, Massova I, Reyes C, Kuhn B, Huo S, Chong L, Lee M, Lee T, Duan Y, Wang W, Donini O, Cieplak P, Srinivasan J, Case DA, Cheatham TE III . Acc Chem Res. 2000; 33:889-97. [PubMed: 11123888]

16. Carrington PE, Chivers PT, Al-Mjeni F, Sauer RT, Maroney MJ. Nat Struct Biol. 2003; 10:126130. [PubMed: 12524532]

17. Leitch S, Bradley MJ, Rowe JL, Chivers PT, Maroney MJ. J Am Chem Soc. 2007; 129:50855095. [PubMed: 17397155]

18. Phillips CM, Schreiter ER, Guo Y, Wang SC, Zamble DB, Drennan CL. Biochemistry. 2008; 47:1938-1946. [PubMed: 18193897]

19. Harding MM. Acta Crystallogr D: Biol Crystallogr. 2002; 58(Pt. 5):872-874. [PubMed: 11976508]

20. Rulisek L, Vondrasek J. J Inorg Biochem. 1998; 71:115-127. [PubMed: 9833317]

21. Ray WJ, Post CB, Liu Y, Rhyu GI. Biochemistry. 1993; 32:48-57. [PubMed: 8418859]

22. Tsai LC, Sjolin L, Langer V, Bonander N, Karlsson BG, Vanngard T, Hammann C, Nar H. Acta Crystallogr D: Biol Crystallogr. 1995; 51:711-717. [PubMed: 15299800] 
23. Nicholls A, Sharp KA, Honig B. Proteins: Struct, Funct Genet. 1991; 11:281-296. [PubMed: 1758883]

24. Sharp KA, Nicholls A, Friedman R, Honig B. Biochemistry. 1991; 30:9686-9697. [PubMed: 1911756]

25. Stultz CM, Karplus M. Proteins: Struct, Funct Genet. 2000; 40:258-289. [PubMed: 10842341]

26. Moreira IS, Fernandes PA, Ramos MJ. Proteins: Struct, Funct Bioinf. 2006; 63:811-821.

27. Spector S, Sauer RT, Tidor B. J Mol Biol. 2004; 340:253-261. [PubMed: 15201050]

28. Floris F, Persico M, Tani A, Tomasi J. Chem Phys. 1995; 195:207-220.

29. Boresch S, Archontis G, Karplus M. Proteins: Struct, Funct Genet. 1994; 20(1):25-33. [PubMed: 7824520]

30. Wang SC, Dias AV, Bloom SL, Zamble DB. Biochemistry. 2004; 43:10018-10028. [PubMed: 15287729]

31. Gomer R, Tryson G. J Chem Phys. 1977; 66:4413-4424.

32. Berman H, Henrick K, Nakamura H. Nat Struct Biol. 2003; 10:980. [PubMed: 14634627]

33. MacKerell AD, Nilsson L. Curr Opin Struct Biol. 2008; 18:194-199. [PubMed: 18281210]

34. Grochowski P, Trylska J. Biopolymers. 2008; 89:93-113. [PubMed: 17969016]

35. Dosanjh NS, Hammerbacher NA, Michel SL. Biochemistry. 2007; 46:2520-2529. [PubMed: 17291009]

36. Dosanjh NS, West AL, Michel SL. Biochemistry. 2009; 48:527-536. [PubMed: 19119856]

37. Emsley P, Crowtan K. Acta Crystallogr. 2004; D60:2126-2132.

38. Brooks BR, Bruccoleri RE, Olafson BD, States DJ, Swaminathan S, Karplus M. J Comput Chem. 1983; 4:187-217.

39. Foloppe N, Sagemark J, Nordstrand K, Berndt KD, Nilsson L. J Mol Biol. 2001; 310:449-470. [PubMed: 11428900]

40. Ryckaert J-P, Ciccotti G, Berendsen HJC. J Comput Phys. 1977; 23:327-341.

41. Brooks CL, Brunger A, Karplus M. Biopolymers. 1985; 24:843-865. [PubMed: 2410050]

42. MacKerell, A.; Nilsson, L. Nucleic Acid Simulations. In: Becker, O.; MacKerell, A.; Roux, B.; Wantanabe, M., editors. Computational Biochemistry and Biophysics. Marcel Dekker, Inc; New York: 2001. p. 441-464.

43. Brooks CL, Karplus M. J Chem Phys. 1983; 79:6312-6325.

44. Hoover WG. Phys Rev A. 1985; 31:1695-1697. [PubMed: 9895674]

45. Young MA, Ravishanker G, Beveridge DL. Biophys J. 1997; 73:2313-2336. [PubMed: 9370428]

46. Beglov D, Roux B. J Chem Phys. 1994; 100:9050-9063.

47. Tsui V, Radhakrishnan I, Wright PE, Case DA. J Mol Biol. 2000; 302:1101-1117. [PubMed: 11183777]

48. Stote RH, Karplus M. Proteins: Struct, Funct Genet. 1995; 23:12-31. [PubMed: 8539245]

49. Grottesi A, Ceruso MA, Colosimo A, Di Nola A. Proteins: Struct Funct and Genet. 2002; 46:287294. [PubMed: 11835504]

50. Stultz CM, Edelman ER. Biophys J. 2003; 85:2198-2204. [PubMed: 14507685] 

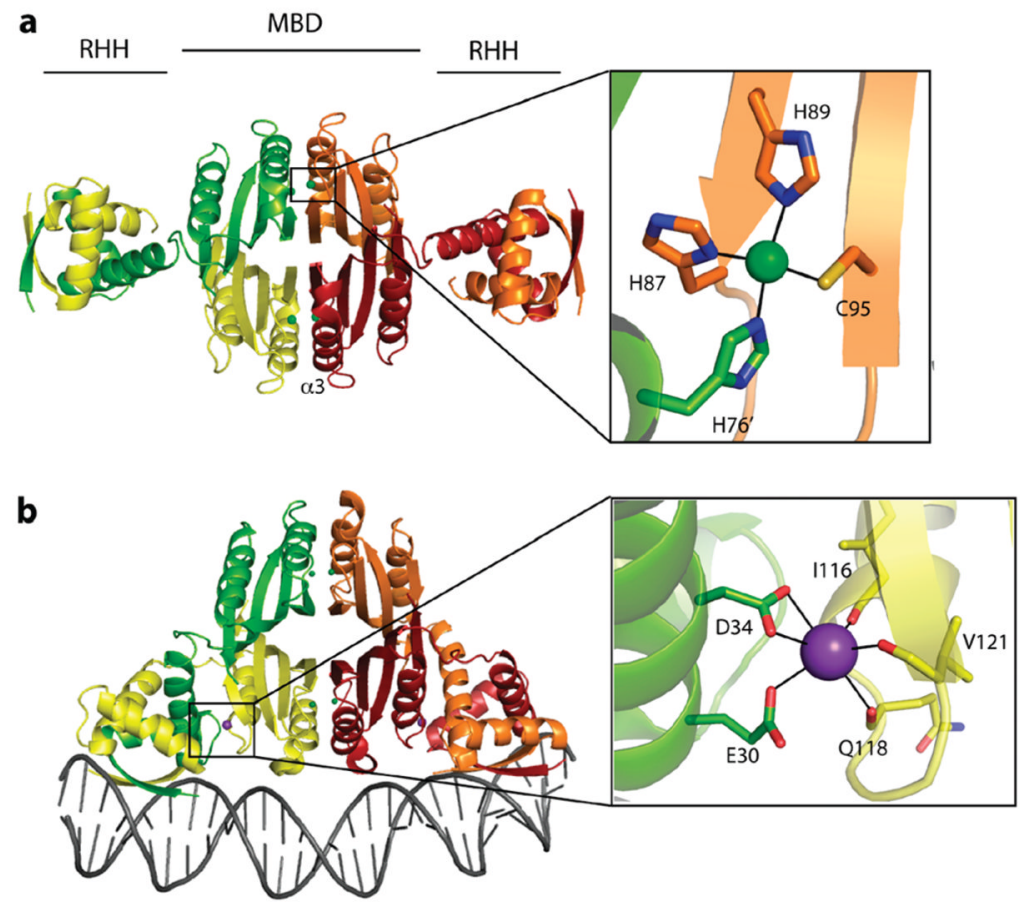

Figure 1.

Crystal structures of $E c N i k R$ and the metal-binding sites. Each of the four monomers is colored individually, and nickel ions are represented with green spheres and potassium ions with purple spheres. (a) $\mathrm{Ni}^{2+}$-bound $E c \mathrm{NikR}$ (PDB $2 \mathrm{HZA}$ ) and the square-planar highaffinity nickel-binding site. (b) EcNikR-DNA complex (PDB 2HZV) and the second metalbinding site with potassium bound in an octahedral geometry. 
a
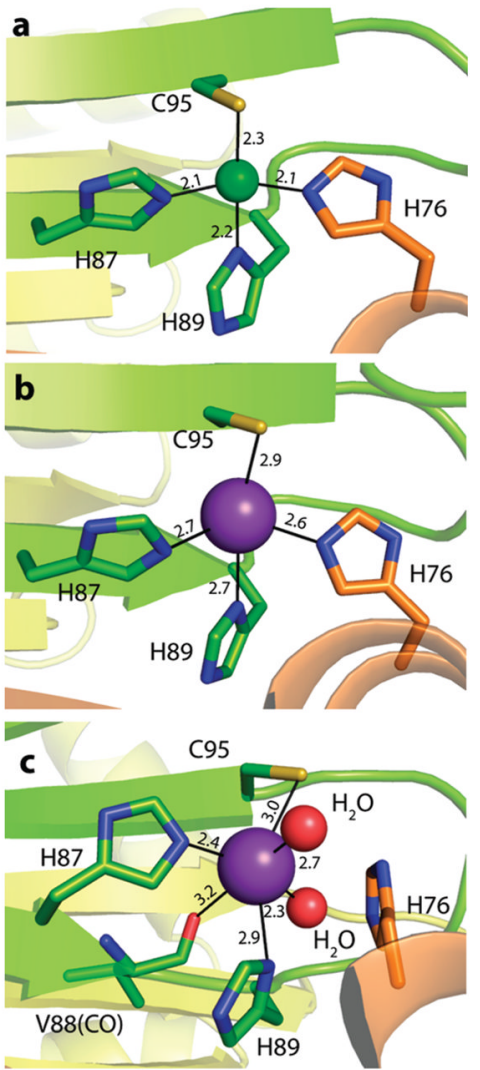

Figure 2.

Representative structures of the high-affinity metal-binding sites after energy minimization and MD simulation studies. High-affinity site with either (a) $\mathrm{Ni}^{2+}$ or (b) $\mathrm{K}^{+}$after energy minimization. Metal-ligand distances are shown in $\AA$. (c) Average structure from MD simulation of high-affinity site containing $\mathrm{K}^{+}$. Coloring is the same as in Figure 1. Water molecules are represented as red spheres. 

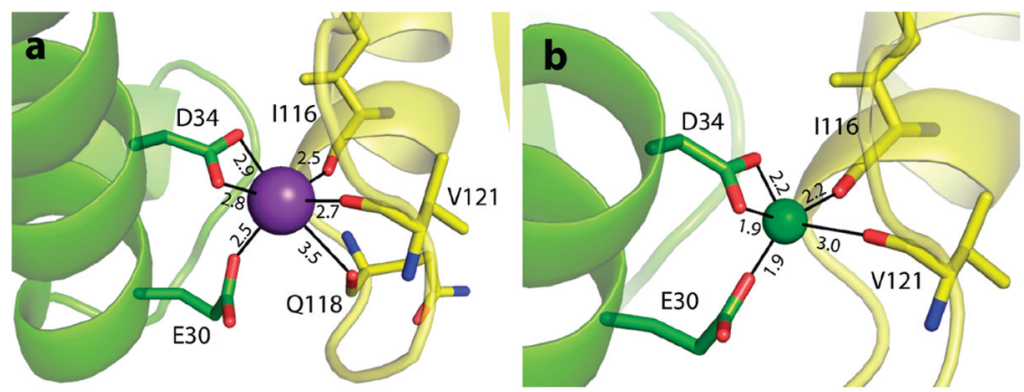

Figure 3.

Representative structures of the second metal-binding site from minimization studies of the EcNikR-DNA complex with either (a) $\mathrm{K}^{+}$or (b) $\mathrm{Ni}^{2+}$ bound in the second metal-binding site. Distances are in $\AA$, and coloring is the same as in Figure 1 . 


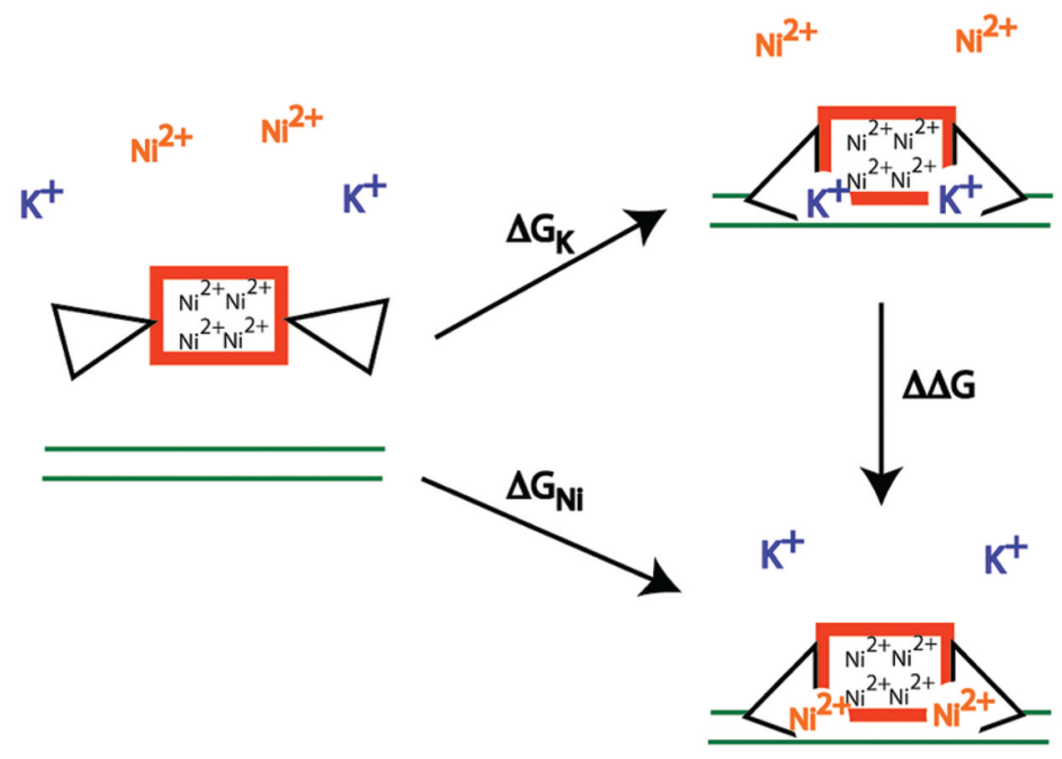

Figure 4.

Thermodynamic path comparing $E c$ NikR-DNA binding with either $\mathrm{K}^{+}\left(\Delta G_{\mathrm{K}}\right)$ or Ni2+ $\left(\Delta G_{\mathrm{Ni}}\right)$ at the second metal-binding site. Red rectangles represent the MBD, black triangles the RHH domains, and parallel green lines the DNA, and ions are explicitly shown in blue or orange. The ions not bound to the structure are infinitely distant from the protein. 


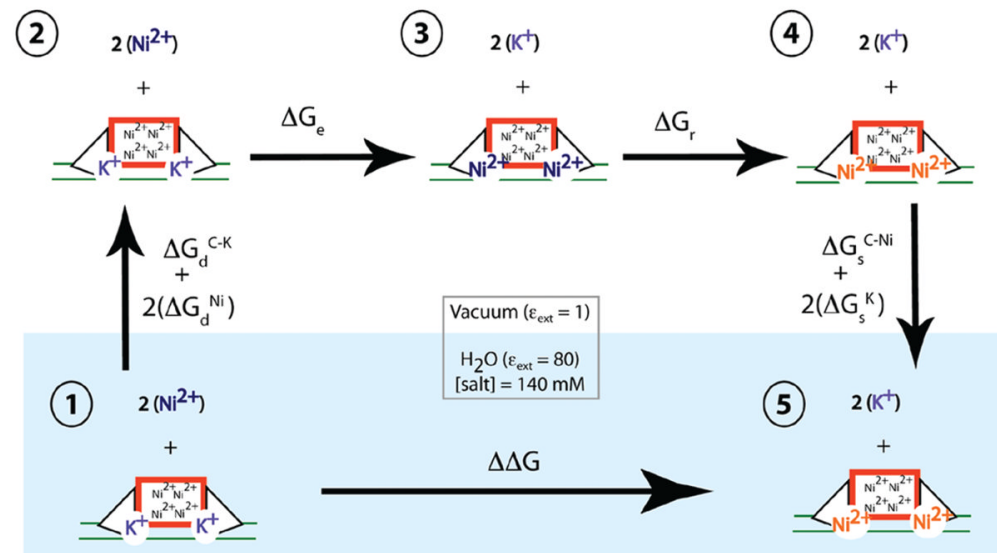

Figure 5.

Thermodynamic cycle for calculating the electrostatic free energy difference associated with replacing potassium with nickel in the second metal-binding site. Numbers in circles delineate each structure in the pathway. Red rectangles represent the MBD, black triangles the RHH domains, and parallel green lines the DNA, and $\mathrm{K}^{+}$ions are explicitly shown in purple. The $\mathrm{Ni}^{2+}$ ions are shown in either blue or orange, depending on the geometry of the groups that surround the ion. In structure $3, \mathrm{Ni}^{2+}$ is placed in the secondary site; however, the coordinating species in the site are arranged in a manner that is consistent with a bound $\mathrm{K}^{+}$ion $\left(\mathrm{Ni}^{2+}\right.$ colored blue). In structure 4 , the coordinating residues have been energy minimized, yielding a rearranged site that has $\mathrm{Ni}^{2+}$-preferred geometry and bond distances $\left(\mathrm{Ni}^{2+}\right.$ colored orange). 


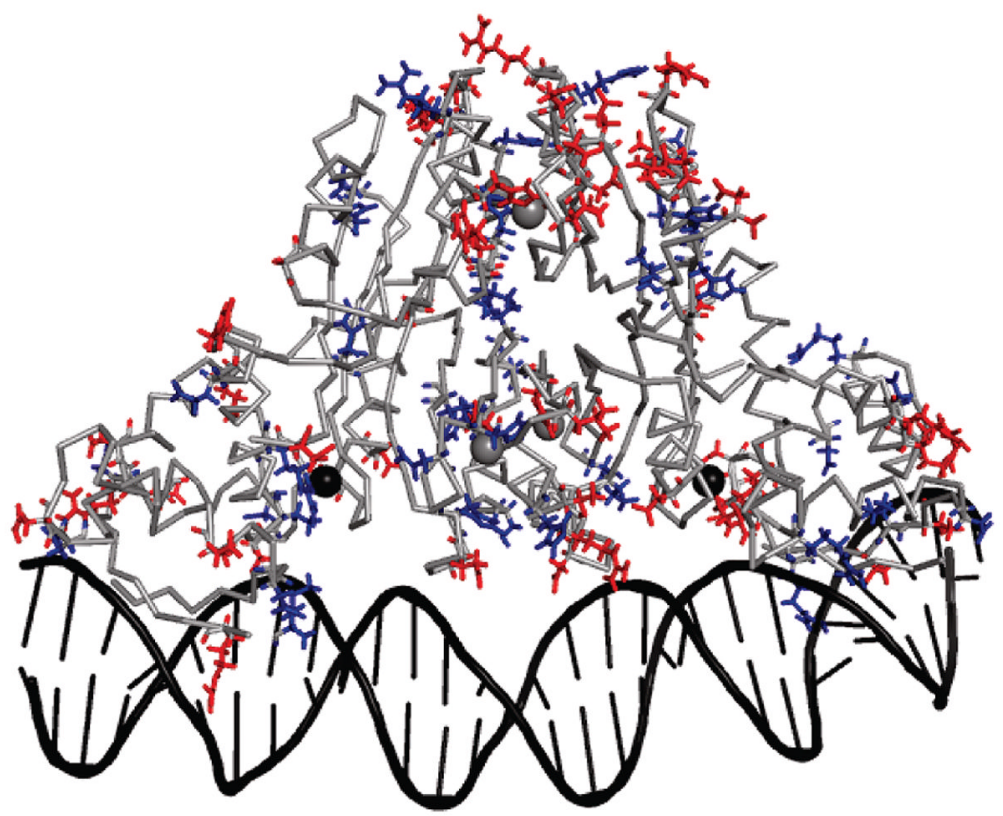

Figure 6.

$E c$ NikR-DNA structure, with residues with the most extreme contributions to $\Delta \Delta G$ colored in red and blue. Red residues contribute $\leftarrow 10 \mathrm{kcal} / \mathrm{mol}$ to $\Delta \Delta G$ and prefer $\mathrm{Ni}^{2+}$ ions in the second metal-binding sites (black spheres). Residues in blue contribute $\geq 10 \mathrm{kcal} / \mathrm{mol}$ to $\Delta \Delta G$ and prefer $\mathrm{K}^{+}$ions in the second metal-binding sites. All backbone atoms are shown in ribbon form and are colored gray. Residues that contribute between -10 and $10 \mathrm{kcal} / \mathrm{mol}$ to the $\Delta \Delta G$ are not shown in stick form. 


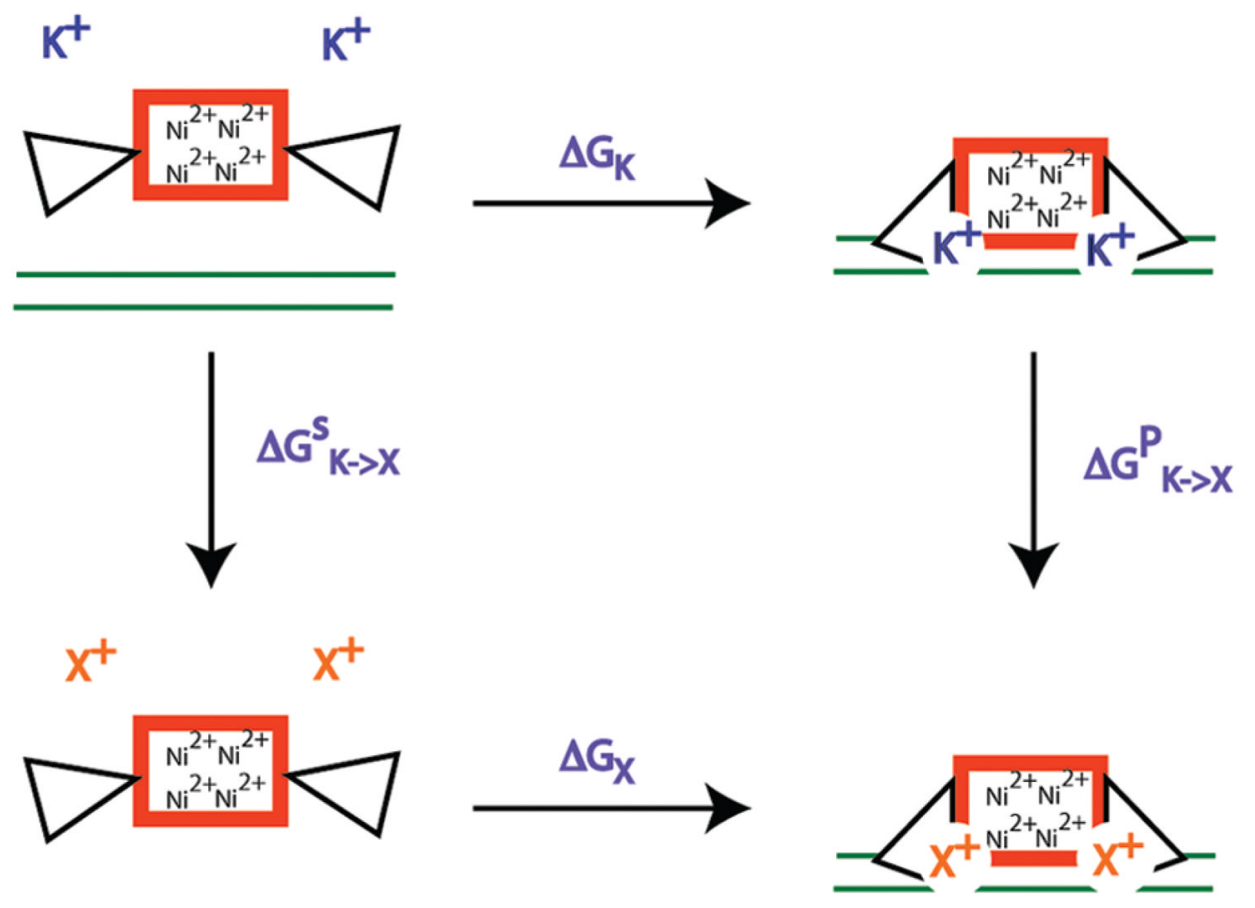

Figure 7.

Thermodynamic cycle for calculating the free energy difference associated with replacing a monovalent cation with the radius of potassium with a cation of larger or smaller radius. NikR representations are the same as in Figure 4, and " $\mathrm{X}^{+}$" represents an ion having different Lennard-Jones parameters than $\mathrm{K}^{+}$. 


\section{Table 1}

Contributions to the Electrostatic Free Energy Difference for the Path Shown in Figure 5

\begin{tabular}{lr}
\hline contribution & value, $\mathbf{k c a l}_{\mathbf{~ m o l}}^{\mathbf{- 1}} \mathbf{s i t e}^{\mathbf{- 1}}$ \\
\hline$\Delta G_{\mathrm{d}}^{\mathrm{C}-\mathrm{K}}+\Delta G_{\mathrm{s}}^{\mathrm{C}-\mathrm{Ni}}$ & 932 \\
$\Delta G_{\mathrm{d}}^{\mathrm{Ni}}$ & 456 \\
$\Delta G_{\mathrm{e}}$ & \\
$\Delta G_{\mathrm{r}}$ & -501 \\
$\Delta G_{\mathrm{s}}^{\mathrm{K}}$ & -61 \\
$\Delta \Delta G$ & -93 \\
\hline
\end{tabular}




\section{Table 2}

Free Energy Differences between EcNikR-DNA Complex with $\mathrm{K}^{+}$and Other Monovalent Ions Bound in the Second Metal-Binding Site (Energies in kcal/mol)

\begin{tabular}{lll}
\hline & $\mathbf{N a}^{+}$ & $\mathbf{C s}^{+}$ \\
\hline$\Delta \Delta G_{\mathrm{vdW}}$ & 4.02 & 0.41 \\
$\Delta \Delta G_{\varepsilon}$ & 1.05 & 0.45 \\
$\Delta \Delta G_{\mathrm{tot}}$ & 5.07 & 0.86 \\
\hline
\end{tabular}

